\title{
Text messaging research participants as a follow-up strategy to decrease emergency department study attrition
}

\author{
Catherine Varner, MD, MSc ${ }^{* \ddagger}$; Shelley McLeod, $\mathrm{PhD}(\mathrm{c}), \mathrm{MSc}^{* 3 \neq}$; Negine Nahiddi, $\mathrm{MD}^{*}$; \\ Bjug Borgundvaag, MD, PhD* ${ }^{\dagger \ddagger}$
}

\section{ABSTRACT}

Objective: Collecting patient-reported follow-up data for prospective studies in the emergency department (ED) is challenging in this minimal continuity setting. The objective of this study was to determine whether text messaging study participants involved in an ongoing randomized trial resulted in a lower rate of attrition as compared to conventional telephone follow-up.

Methods: This was a nested cohort analysis of research participants enrolled in a randomized controlled trial assessing head injury discharge instructions. During the first 4 months of study follow-up, participants were contacted by a conventional telephone call. For the final 3 months, participants were contacted by text messaging following the first failed telephone attempt.

Results: A total of 118 patients were enrolled in the study (78 underwent conventional follow-up, and 40 received text messages). During the period of conventional follow-up, 3 participants withdrew from the study. Of the remaining 75 participants, $24(32.0 \%)$ at 2 weeks and $32(42.7 \%)$ at 4 weeks were unable to be contacted. Of the 40 participants receiving a reminder text message, $4(10.0 \%)$ at 2 weeks and $10(25.0 \%)$ at 4 weeks were unable to be contacted. Overall, text messaging study participants decreased attrition by $22 \%$ (95\% Cl: $5.9 \%, 34.7 \%)$ and $17.7 \%(95 \% \mathrm{Cl}:-0.8 \%, 33.3 \%)$ at 2- and 4-week follow-ups, respectively.

Conclusions: In this ED cohort participating in a randomized trial, text message reminders of upcoming telephone followup interviews decreased the rate of attrition. Text messaging is a viable, low-cost communication strategy that can improve follow-up participation in prospective research studies.

\section{RÉSUMÉ}

Introduction: La collecte de données sur le suivi des patients en vue d'études prospectives au service des urgences (SU) présente des difficultés dans un contexte de continuité minimale. L'étude décrite ici avait pour but de déterminer si l'envoi de messages textes (textos) à des participants engagés dans un essai à répartition aléatoire en cours permettrait une diminution du taux d'abandon comparativement au suivi téléphonique habituel.

Méthode: II s'agit d'une analyse de cohorte nichée, menée dans le cadre d'un essai comparatif, à répartition aléatoire, visant à évaluer les instructions données au moment du congé de I'hôpital à des patients ayant subi un traumatisme crânien. Durant les 4 premiers mois de suivi, les participants ont été joints par téléphone. Durant les 3 derniers mois, on communiquait avec les participants par des messages textes dès l'échec de la première tentative de communication téléphonique.

Résultats: Au total, 118 patients ont été sélectionnés (78 ont fait l'objet du suivi habituel et 40 ont reçu des messages textes). Durant la période de suivi habituel, 3 participants se sont retirés de l'étude. Sur les 75 participants restants, $24(32,0 \%)$ n'ont pu être joints au bout de 2 semaines et $32(42,7 \%)$, au bout de 4 semaines. Quant aux 40 participants qui ont reçu des messages textes de rappel, 4 (10,0\%) au bout de 2 semaines et $10(25,0 \%)$ au bout de 4 semaines n'ont pu être joints. Dans l'ensemble, l'envoi de messages textes aux participants à l'étude a permis d'abaisser le taux d'abandon de $22 \%$ (IC à $95 \%$ : 5,9\%-34,7 \%) et de 17,7 \% (IC à $95 \%$ : -0,8 \%-33,3\%) au bout de 2 semaines et de 4 semaines de suivi, respectivement.

Conclusions: Dans cette cohorte de patients au SU qui participaient à un essai à répartition aléatoire, les rappels par message texte concernant des entretiens de suivi téléphonique à venir ont permis d'abaisser le taux d'abandon. La messagerie texte s'avère donc une solution de communication viable et peu coûteuse, susceptible d'améliorer le suivi des participants à des études de recherche prospectives.

Keywords: text messaging, study attrition, trial design

From the *Department of Family and Community Medicine, University of Toronto, Toronto, ON; †Department of Emergency Medicine, Mount Sinai Hospital, Toronto, ON; and ¥Schwartz/Reisman Emergency Medicine Institute, Mount Sinai Hospital, Toronto, ON.

Correspondence to: Dr. Catherine Varner, Mount Sinai Hospital, Division of Emergency Medicine, 600 University Avenue, Rm. 206, Toronto, ON M5G 1X5; Email: Catherine.Varner@sinaihealthsystem.ca 


\section{INTRODUCTION}

Text messaging is ubiquitous in modern communication and has been widely adopted by the general population. When considering other modes of communication, text messaging is efficient, mobile, low cost, and has the advantage of instant transmission. ${ }^{1}$ Text messaging has become the most used method of daily communication between family and friends, overtaking speaking on a mobile phone. ${ }^{2}$ Text messages are also perceived as being less invasive to daily lives than phone calls and more convenient for exchanging information. ${ }^{3}$ This method of communication has been effective in the health care setting to provide appointment reminders, facilitate self-management of chronic illnesses, and encourage medication adherence. ${ }^{4-6}$ Several reviews of the literature have illustrated wide application and potential for mobile phones to increase access to health care; enhance the efficiency of service delivery; improve diagnosis, treatment, and rehabilitation; and support public health. ${ }^{4-6}$

Despite the widespread application of text messaging to facilitate health care delivery, there is a paucity in the literature supporting the use of text messaging to facilitate clinical research, specifically to assist in research participant follow-up. ${ }^{7}$ Incomplete or missing outcome data from patients lost to follow-up or patients who withdraw from study participation present a major threat to the internal and external validity of a clinical trial. The study results may be biased if patients are unable to follow up because of factors related to the outcome of interest. ${ }^{8}$ Therefore, researchers should anticipate and plan a pragmatic approach to decrease study attrition at the stage of trial design and patient accrual. Text messaging, proven effective in the health care delivery setting, offers a novel means to improve clinical research study attrition.

The objective of this study was to determine whether text messaging study participants involved in an ongoing randomized controlled trial (RCT) resulted in a lower rate of attrition compared to conventional telephone follow-up.

\section{METHODS}

This was a nested cohort analysis of research participants enrolled in an RCT assessing minor traumatic brain injury (MTBI) discharge instructions. The trial took place in the emergency department (ED) of an academic tertiary care hospital in Toronto, Ontario (annual census 55,000) from October 2014 to May 2015. Patients ages 18 to 64 years presenting to the ED with a Canadian Emergency Department Information System (CEDIS) chief complaint of a head injury or suspected concussion occurring within 24 hours were eligible for study inclusion. ED nurses screened potential patients, and once eligibility was confirmed by the attending physician, informed written consent was obtained from trained research personnel (when available) or by the emergency physician or nurse. Patients were excluded if they had an acute intracranial injury identified on a head computed tomography (including but not limited to subarachnoid hemorrhage, skull fracture, intracranial contusion, or epidural hematoma), a Glasgow Coma Scale score of $<15$, were cognitively impaired, did not speak English, or did not have a telephone. The study protocol was approved by the institutional research ethics board (REB).

Patients were contacted by telephone at 2 weeks and 4 weeks following their index ED visit to repeat a symptom questionnaire and document other MTBIrelated outcomes. During the first 4 months of study follow-up, participants were contacted by a conventional telephone call only. If telephone contact was not successful on the first day of the scheduled follow-up, telephone attempts were made on 2 more days. Because of participant attrition at the study's midpoint, REB approval was applied for and received to send text message reminders to patients regarding their upcoming telephone calls. The revised consent form asked patients if they were willing to be contacted by a text message in the event that telephone contact was not successful on the first day of the scheduled follow-up. Research personnel were provided a study-specific mobile phone to both send and receive text messages to participants. Text messages were sent and responded to, if applicable, during daytime hours on Monday to Friday. Study participants were not reimbursed for any charges that they may have incurred for the text messages. If contact was not made after three telephone attempts and a text communication, patients were considered lost to follow-up (Figure 1). No other changes to the protocol were made during the study period. The text message was structured as follows: "Hi [name of study participant]. Recently you enrolled in a research study at Mount Sinai Hospital. A research assistant will be calling you today to complete a questionnaire. Please note that this will be a blocked number. If we are unable to get in touch today, please suggest to us a better time to call, or call 416586-4800, ext. 5959 to speak to the research assistant. Thank you for your participation." No personal health information was exchanged in the text messages. 


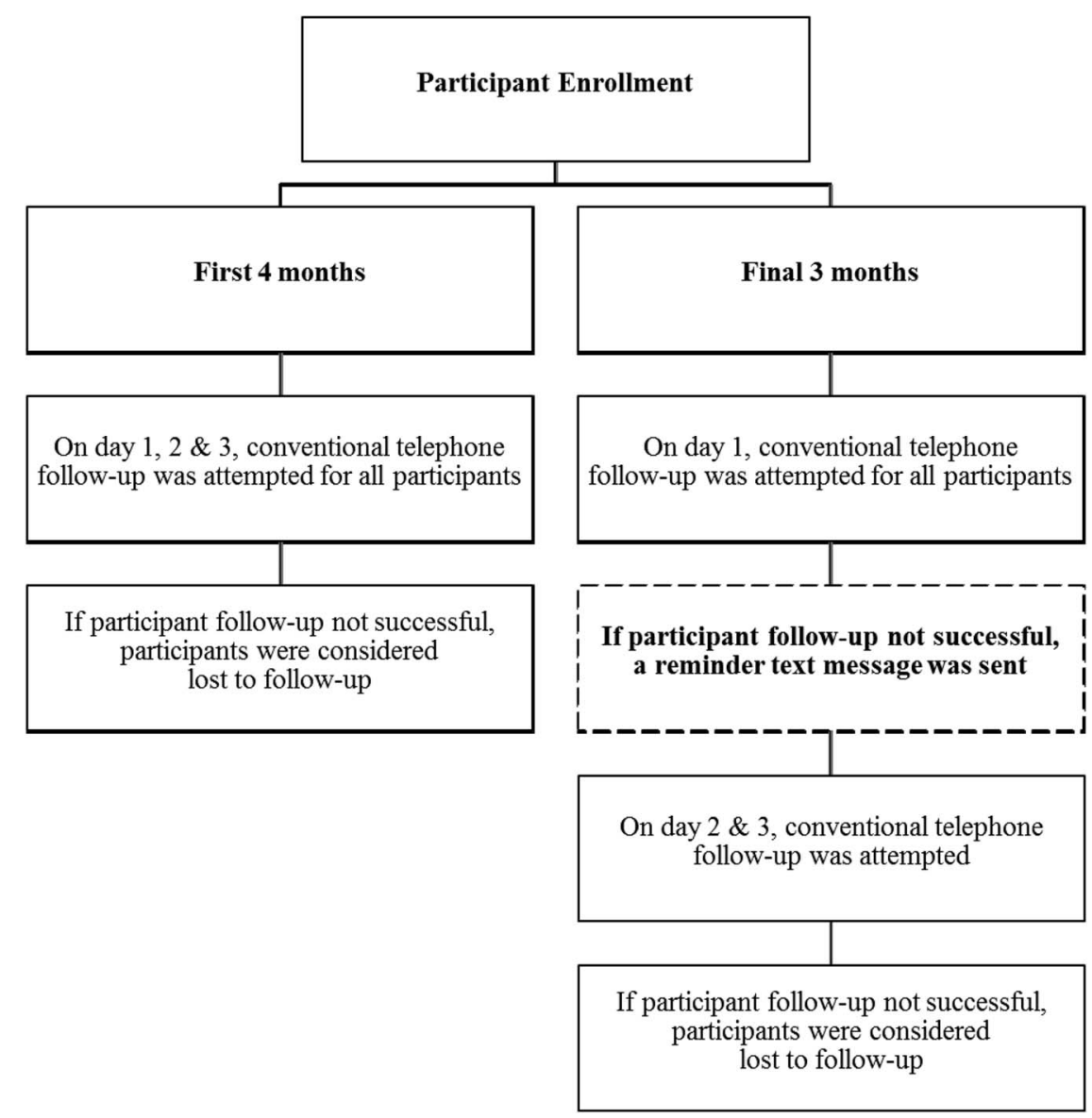

Figure 1. Follow-up methods for enrolled patients.

The primary outcome was the proportional difference in loss to a follow-up at 2 weeks between the group receiving text messages and those receiving conventional telephone follow-up. The difference in loss to follow-up at 4 weeks was also measured, and anecdotal experiences were noted. Data were entered directly into a studyspecific Microsoft Excel database (Microsoft Corporation, Redmond, Washington). Descriptive statistics were summarized using means and standard deviations or proportional differences with $95 \%$ confidence intervals where appropriate. Loss to follow-up between the group receiving texts and the group not receiving texts was compared using a Fisher exact test or chi-square test, where appropriate. All statistical analyses were conducted using SAS (v.9.3, SAS Institute, Cary, North Carolina).

\section{RESULTS}

A total of 118 patients were enrolled in the study (40 received text messages, and 78 underwent conventional follow-up). The mean age was 35.2 (SD 13.7) years, and
$43(36.4 \%)$ were male. Patient characteristics between the groups were similar (Table 1$)$.

During the period of conventional follow-up, three participants withdrew from the study. Of the remaining 75 participants, $24(32.0 \%)$ at 2 weeks and $32(42.7 \%)$ at 4 weeks were unable to be contacted. Of the 40 participants receiving a reminder text message, 4 (10.0\%) at 2 weeks and $10(25.0 \%)$ at 4 weeks were unable to be contacted. Overall, text messaging study participants decreased attrition by $22 \%$ (95\% CI: $5.9 \%, 34.7 \%$ ) and $17.7 \%(95 \% \mathrm{CI}:-0.8 \%, 33.3 \%)$ at 2 - and 4-week follow-ups, respectively (Figure 2).

Although not formally captured, patients often replied to text messages with a request to reschedule their follow-up telephone interview to a more convenient time, often to not interrupt work obligations. Two examples of these scheduling request text messages are as follows:

"I am working now. Can I call you at noon?"

"Just about to step into a meeting at 9:15. I am free at 10:15. You can call at that time." 
Table 1. Baseline characteristics of enrolled patients

\begin{tabular}{lccc}
\hline & $\begin{array}{c}\text { Received text message } \\
(\mathrm{n}=40)\end{array}$ & $\begin{array}{c}\text { Did not receive text message } \\
(\mathrm{n}=78)\end{array}$ & $\begin{array}{c}\text { Total } \\
(\mathrm{n}=118)\end{array}$ \\
\hline Mean (SD) age (years) & $36.2(14.4)$ & $34.7(13.5)$ & $35.2(13.7)$ \\
Male & $20(50.0 \%)$ & $23(29.5 \%)$ & $43(36.4 \%)$ \\
Head CT & $9(22.5 \%)$ & $22(28.2 \%)$ & $31(26.3 \%)$ \\
Ambulance & $12(30.0 \%)$ & $23(29.5 \%)$ & $35(29.7 \%)$ \\
Headache & $18(45.0 \%)$ & $46(59.0 \%)$ & $64(54.2 \%)$ \\
Nausea & $9(22.5 \%)$ & $26(33.3 \%)$ & $35(29.7 \%)$ \\
Dizziness & $6(15.0 \%)$ & $26(33.3 \%)$ & $32(27.1 \%)$ \\
Visual complaints & $9(22.5 \%)$ & $13(16.7 \%)$ & $22(18.6 \%)$ \\
Analgesia given & $15(37.5 \%)$ & $26(33.3 \%)$ & $41(34.8 \%)$ \\
Antiemetic given & $1(2.5 \%)$ & $6(7.7 \%)$ & $7(5.9 \%)$ \\
History of anxiety/depression & $7(17.5 \%)$ & $8(10.3 \%)$ & $15(12.7 \%)$ \\
CTAS 1 & $1(2.5 \%)$ & 0 & $1(0.9 \%)$ \\
CTAS 2 & $2(5.0 \%)$ & $7(9.0 \%)$ & $9(7.6 \%)$ \\
CTAS 3 & $20(50.0 \%)$ & $42(53.85 \%)$ & $62(52.5 \%)$ \\
CTAS 4 & $15(37.5 \%)$ & $27(34.6 \%)$ & $42(35.6 \%)$ \\
Mechanism of injury & & & \\
$\quad$ Assault & $3(7.5 \%)$ & $5(6.4 \%)$ & $8(6.8 \%)$ \\
Sports-related & $7(17.5 \%)$ & $3(3.9 \%)$ & $10(8.5 \%)$ \\
MVC or bike & $7(17.5 \%)$ & $24(30.8 \%)$ & $31(26.3 \%)$ \\
Fall & $14(35.0 \%)$ & $12(15.4 \%)$ & $48(40.7 \%)$ \\
Other & $9(22.5 \%)$ & $21(17.8 \%)$ \\
\hline CT = computed tomography; CTAS $=$ Canadian $)$ & Triage and Acuity Scale; MVC motor vehicle crash; SD = standard deviation.
\end{tabular}

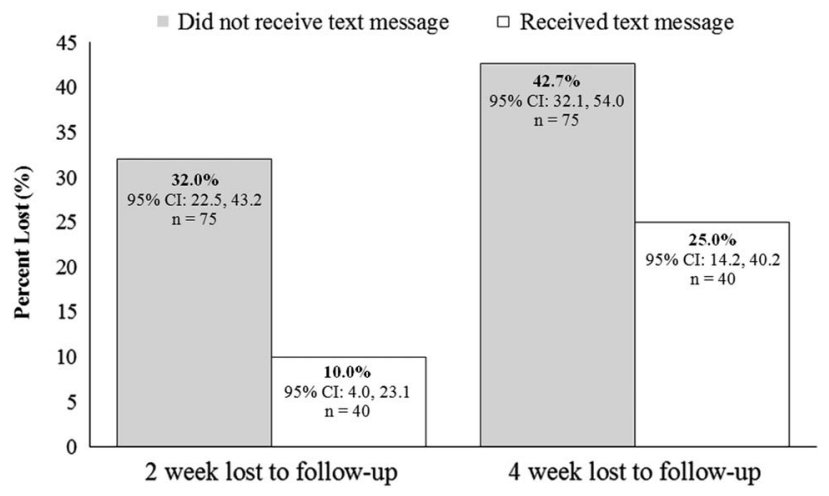

Figure 2. The proportion of patients lost to follow-up at 2 and 4 weeks between those who received conventional telephone follow-up compared to those receiving text messages. Text messaging decreased attrition by $22 \%$ (95\% Cl: $5.9 \%, 34.7 \%$ ) and $17.7 \%$ (95\% Cl: $-0.8 \%, 33.3 \%)$ at 2 and 4 week follow-up, respectively.

Additionally, patients expressed preferences towards text messaging, as one patient responded, "It's better you text. It's more efficient. I will call [the research assistant] today."

\section{DISCUSSION}

The primary objective of this study was to determine whether text messaging study participants involved in an ongoing randomized trial resulted in a lower rate of attrition compared to conventional telephone follow-up. Our findings in this ED cohort suggest that text message reminders of upcoming telephone follow-ups decreased the rate of attrition, resulting in a more efficient study completion and potential for less biased results.

Planning a pragmatic approach to decrease study attrition is important when designing a clinical trial. The failure to account for all included participants at the end of the trial presents a major threat to the internal validity of the study findings. ${ }^{8}$ A recent meta-analysis of three studies assessed the effectiveness of sending electronic mail (email) prompts to 437 trial participants in an effort to improve response rates to postal questionnaires. The authors concluded that the use of electronic prompts increased the response rate $69.5 \%$ in the electronic prompt group $\mathrm{v}$. $60.7 \%$ in the control group; $\Delta 8.8 \%$; $95 \%$ CI: $-0.11 \%$, $17.7 \%$ ) and reduced the time of response (23 days in the electronic prompt group v. 33 days in the control group; hazard ratio $=1.27 ; 95 \%$ CI: $1.11,1.47) .{ }^{9}$ The improved response rates following email reminders are consistent with our findings, and although text messaging and email are different modes of electronic reminders, both are useful in decreasing study attrition.

With respect to text messaging study participants, one study has reported that the effectiveness of text messaging 
pre-notification reminders for postal questionnaires did not improve response rates. Starr et al. ${ }^{10}$ evaluated two interventions aimed at increasing response rates to postal questionnaires within a large RCT in the UK: prenotification through short messenger service (SMS) text prior to sending the initial mailing of trial questionnaires versus no pre-notification; for non-responders to the initial mailing of the questionnaires, an email reminder (containing a hyperlink to complete the questionnaire online) versus a postal reminder. In this $2 \times 2$ factorial trial, participants were randomly assigned to the prenotification comparison or the reminder comparison or both. SMS text pre-notification of a questionnaire delivery and email delivery of questionnaire reminders did not improve response rates. ${ }^{10}$ It is possible that text messaging in our study was more effective because follow-up occurred the same day, using the same telephone number.

Text messaging to improve follow-up for treatment or clinical reassessment has been shown to be effective in the ED setting. Arora et al. ${ }^{11}$ performed an RCT of ED patients with an outpatient follow-up scheduled at the time of an ED discharge. Patients who received an automated text message reminder were more likely to attend their scheduled follow-up visit $(72.6 \%$ in the intervention group compared with $62.1 \%$ in the control group; $\Delta 10.5 \%$; $95 \%$ CI: $0.3 \%, 20.8 \%$; number needed to treat 9.5). ${ }^{11}$ Wolff et al. ${ }^{12}$ conducted an RCT of adolescent females diagnosed with pelvic inflammatory disease, which found that patients who received standard discharge instructions plus text message reminders were more likely to receive recommended follow-up care within 72 hours of the ED discharge compared to patients who received standard discharge instructions (relative risk 2.9, 95\% CI: 1.4, 5.7; number needed to treat 4, 95\% CI: 1.4, 5.7). ${ }^{12}$ These ED-specific findings are consistent with previous studies demonstrating improved follow-up adherence in other health care settings when text messaging was used. ${ }^{6}$

This study is not without limitations. The main limitation of this study is that it is a nested cohort analysis within a small RCT conducted at a single academic centre. These findings may not be generalizable to other centres. Future studies should investigate text messaging as a means to decrease study attrition as the focus of the investigation. It is possible that unmeasured confounders (i.e., socioeconomic and employment status) contributed to the differences in loss to follow-up between the two groups. This study did not address other methods that could be used to mitigate loss to follow-up, such as monetary incentives, which have been previously shown to improve response rates. ${ }^{7}$ However, the use of monetary incentives carries both practical and ethical considerations, particularly in the context of a clinical trial. Given that email has also been effective at decreasing study attrition for postal questionnaires, email may be another effective, low-cost option to use going forward. ${ }^{10}$ However, text messaging is an easily-adopted, methodological intervention that can be implemented using a low-cost data mobile phone plan.

\section{CONCLUSIONS}

Overall, few strategies in randomized trials have been investigated to reduce loss to follow-up. In our ED cohort participating in a randomized trial, text message reminders of upcoming telephone follow-ups decreased the rate of attrition. Our findings suggest that text messaging is an easily-adopted, low-cost technique that can improve study participation and follow-up, particularly in the low-continuity ED setting.

Competing interests: None declared. This study was funded by the College of Family Physicians of Canada Janus Research Grant Training Level 1.

\section{REFERENCES}

1. Purcell K, Entner R, Henderson N. Pew Research Center's Internet and American Life Project. Cell phone activities; 2012. Available at: http://www.pewinternet.org (accessed 2 May 2016).

2. Smith A. Pew Research Center's Internet and American Life Project. U.S. smartphone use in 2015. Available at: http://www.pewinternet.org (accessed 2 May 2016).

3. Kaplan WA. Can the ubiquitous power of mobile phones be used to improve health outcomes in developing countries? Global Health 2006;23(2):9.

4. Car J, Gurol-Urganci I, de Jongh T, et al. Mobile phone messaging reminders for attendance at healthcare appointments. Cochrane Database Syst Rev 2012;7:CD007458.

5. de Jongh T, Gurol-Urganci I, Vodopivec-Jamsek V, et al. Mobile phone messaging for facilitating self-management of long-term illnesses. Cochrane Database Syst Rev 2012;12: CD007459.

6. Liu Q, Abba K, Alejandra MM, et al. Reminder systems to improve patient adherence to tuberculosis clinic appointments for diagnosis and treatment. Cochrane Database Syst Rev 2014;11:CD006594.

7. Brueton VC, Tierney J, Stenning S, et al. Strategies to improve retention in randomized trials. Cochrane Database Syst Rev 2013;12:MR000032, doi:10.1002/14651858. MR000032.pub2. 
8. Akl EA, Briel M, You JJ, et al. LOST to follow-up Information in Trials (LOST-IT): a protocol on the potential impact. Trials 2009;10:40.

9. Clark L, Ronaldson S, Dyson L, et al. Electronic prompts significantly increase response rates to postal questionnaires: a randomized trial within a randomized trial and meta-analysis. 7 Clin Epidemiol 2015;68(12):1446-50.

10. Starr K, McPherson G, Forrest M, et al. SMS text pre-notification and delivery of reminder emails to increase response rates to postal questionnaires in the SUSPEND trial: a factorial design, randomised controlled trial. Trials 2015;16:295.

11. Arora S, Burner E, Terp S, et al. Improving attendance at post-emergency department follow-up via automated reminders: a randomized controlled trial. Acad Emerg Med 2015;22:31-7.

12. Wolff M, Balamuth F, Sampayo E, et al. Improving adolescent pelvic inflammatory disease follow-up from the emergency department: randomized controlled trial with text messages. Annals Emerg Med 2016;67(5):602-9. 